\title{
PAISAJE BIOCULTURAL Y TERRITORIO, REFLEXIONES SOBRE LA GESTIÓN DEL PATRIMONIO DEL "PAISAJE AGAVERO Y ANTIGUAS INSTALACIONES DEL TEQUILA"
}

\section{BIOCULTURAL LANDSCAPE AND TERRITORY, REFLECTIONS ON THE MANAGEMENT OF THE HERITAGE OF THE "AGAVE LANDSCAPE AND ANCIENT INSTALLATIONS OF TEQUILA"}

\author{
ORENDAIN VERDUZCO, Tomás Eduardo \\ Secretaria de Cultura Jalisco \\ Dirección General de Patrimonio Cultural \\ Director General \\ Reforma 425, colonia centro, Guadalajara, Jalisco, C.P. 44100. México \\ Correo electrónico: orendaintome@yahoo.com.mx, tomas.orendain@jalisco.gob.mx \\ Teléfono: +52 (333) 3030-4500 ext. 50068, 54752, 54753 \\ MICHEL PARRA, J. Guadalupe \\ Universidad de Guadalajara (UdeG) \\ Centro Universitario del Sur (CUSUSR) \\ Director General y profesor investigador de tiempo completo, Titular C \\ Av. Enrique Arreola Silva 883, colonia centro, Ciudad Guzmán, Jalisco, C.P. 49000. México \\ Correo electrónico: michelp@cusur.udg.mx \\ Teléfono: +52 (341) 5752222 ext. 46074
}

\section{Resumen}

La declaratoria por la UNESCO del Paisaje Agavero y Antiguas Instalaciones del Tequila, como patrimonio mundial, se obtiene por la extensión territorial de siembra de agave Tequilana Weber (variedad de agave azul), demostrando que por cuatro siglos se ha sembrado por los lugareños de donde se extrae la bebida de tequila, siendo un Paisaje Vivo y Productivo. Dividiéndose en: zona núcleo 1, con una superficie de 34 mil hectáreas aproximadamente, en la que predomina el territorio correspondiente al Volcán de Tequila (montaña, valle y barrancas) y los asentamientos humanos. Está integrada por los municipios de Magdalena, Tequila, Amatitán y Arenal. Dentro del polígono antes mencionado se encuentra la zona núcleo 2 perteneciente a la delimitación del sitio arqueológico de Guachimontones, municipio de Teuchitlán y el tercer polígono o zona de Amortiguamiento con una superficie aproximada de 51 mil hectáreas, predominando el Valle y la Barranca del Rio Santiago, correspondiente a los municipios de Tala, Ahualulco de Mercado, San Juanito de Escobedo y Etzatlán.

La complejidad que implica la puesta en práctica de estas estrategias culturales es el mantener a la población local en su región, evitando la migración, teniendo como resultado el abandono de su tierra. Promover los valores, las prácticas y las tradiciones culturales, además del fomento del conocimiento desarrollado en torno al cultivo de agave y sus derivados que da como resultado un Paisaje Excepcional y único en el Mundo de ahí la importancia de su preservación. 
Sabedores de que las zonas de protección no se dan por sí mismos, debemos promover los valores, las prácticas y las tradiciones culturales, además del desarrollado en torno de las actividades locales. Ya que, si no se cuenta con el apoyo, pero sobre todo la apropiación de la población. Teniendo como principal reto el que las personas que viven de sus actividades cotidianas estén consientes de la responsabilidad que implica el cuidado y evolución de este Paisaje vivo y posteriormente se ligue al turismo y las nuevas tecnologías sin detrimento o de impacto negativo sobre el paisaje. Esto con el objetivo de salvaguardar y preservar el legado cultural, material e inmaterial de la actividad humana en comunión con el medio ambiente, a los que los habitantes de la entidad, por su significado y valor, les atribuyen importancia intelectual, científica, tecnológica, histórica, natural, literaria, artística, arqueológica, antropológica, paleontológica, etnológica, arquitectónica, industrial y urbana, a lo de cada grupo social sin transgredirla, hace que dicha estrategia cobre múltiples significados. En base a que transite y se adapte a las nuevas tecnologías, así como acciones ligadas al Plan Estatal de Acción ante el Cambio Climático a lo largo del territorio en que se encuentra en donde las acciones de mitigación y adaptación son claramente aplicables en pro de mantener eco-sistemas endémicos saludables, en acciones para conservar la biodiversidad del territorio.

\section{Abstract}

The declaration by UNESCO of the Agave Landscape and Ancient Installations of Tequila, as a world heritage, is obtained by the territorial extension of agave Tequilana Weber (variety of blue agave), showing that during four centuries it has been planted by the locals from where the tequila drink is extracted, being a living and productive landscape. It is divided into: core zone 1 , with an area of approximately 34 thousand hectares, in which the territory corresponding to the Tequila Volcano (mountain, valley and ravines) and human settlements predominates. It is integrated by the municipalities of Magdalena, Tequila, Amatitán and Arenal. Within the aforementioned polygon is the core zone 2 pertaining to the delimitation of the archaeological site of Guachimontones, municipality of Teuchitlán and the third polygon or zone of Amortiguamiento with an approximate area of 51 thousand hectares, predominating the Valley and the Ravine of the Santiago River, corresponding to the municipalities of Tala, Ahualulco de Mercado, San Juanito de Escobedo and Etzatlán.

The complexity involved in implementing these cultural strategies is to keep the local population in their region, avoiding migration, resulting in the abandonment of their land. Promoting values, practices and cultural traditions, as well as the promotion of the knowledge developed around the cultivation of agave and its derivatives that results in an Exceptional and Unique Landscape in the World, hence the importance of its preservation.

Knowing that protection zones do not occur on their own, we must promote values, practices and cultural traditions, in addition to those developed around local activities. Because if you do not know how much support, but especially the appropriation of the population. Having as main challenge that people who live from their daily activities are aware of the responsibility involved in the care and evolution of this living landscape and subsequently linked to tourism and new technologies without detriment or negative impact on the landscape. This with the aim of safeguarding and preserving the cultural, material and immaterial legacy of human activity in communion with the environment, to which the inhabitants of the entity, for their meaning and value, attribute to them intellectual, scientific, technological importance, historical, natural, literary, artistic, archaeological, anthropological, paleontological, ethnological, architectural,

Citación: ORENDAIN, T. E. y MICHEL, J. G. Paisaje biocultural y territorio, reflexiones sobre la gestión del patrimonio del "paisaje agavero y antiguas instalaciones del tequila". En: Libro de proceedings, CTV 2018. XII Congreso Internacional Ciudad y Territorio Virtual. "Ciudades y Territorios Inteligentes". UNCuyo, Mendoza, 5-7 septiembre 2018. Barcelona: CPSV, 2018, p. 63-76. 
industrial and urban, to that of each social group without transgressing it, that strategy takes on multiple meanings. Based on the fact that it transits and adapts to new technologies, as well as actions linked to the State Action Plan on Climate Change throughout the territory where it is located, where mitigation and adaptation actions are clearly applicable in order to maintain healthy endemic ecosystems, in actions to conserve the biodiversity of the territory.

\section{La declaratoria por la UNESCO}

En el marco de la XXX Asamblea del Comité del Patrimonio Mundial de la UNESCO celebrado en la ciudad de Vilnius, Lituania el 16 de julio de 2006 se logró la inscripción del sitio denominado: Agave Landscape and Ancient Industrial Facilities of Tequila, registrado con el número de identificación C1209. ${ }^{1}$

De acuerdo a la decisión del Comité del Patrimonio Mundial son cuatro criterios de valor universal excepcional con los que se inscribe el sitio en la Lista del Patrimonio Mundial:

\subsection{Criterio II}

El cultivo del agave y su destilación ha producido un paisaje distintivo dentro del cual se ubica una colección de valiosas haciendas y destilerías que reflejan la fusión de la tradición prehispánica de la fermentación del jugo de mezcal con el proceso europeo de destilación y de tecnologías locales e importadas, ambas europeas y americanas.

\subsection{Criterio IV}

La colección de haciendas y destilerías, en muchos casos completas con su equipamiento y reflejando el crecimiento de la destilación de tequila en el paso de doscientos cincuenta años, son en conjunto un extraordinario ejemplo de complejos arquitectónicos distintivos que ilustran la fusión de tecnologías y culturas.

\subsection{Criterio $V$}

El Paisaje Agavero ejemplifica el vínculo continuo entre la antigua cultura mesoamericana del agave y el presente, así como el continuo proceso de cultivo desde el siglo XVII cuando se establecen las primeras plantaciones intensivas y las destilerías inician la producción de tequila. El paisaje constituido por campos agaveros, destilerías, haciendas y poblados es un ejemplo excepcional de un asentamiento humano tradicional y un uso de la tierra que es representativo de la cultura especifica que se desarrolló en Tequila.

\subsection{Criterio VI}

El Paisaje de Tequila ha generado trabajos literarios, películas, música, arte y danza, todos celebrando los vínculos entre México, el tequila y su lugar de origen en Jalisco. El Paisaje Agavero de Tequila está fuertemente asociado con percepciones de significado cultural más allá de sus fronteras.

El expediente de postulación fue aprobado con beneplácito por los países representados en el

\footnotetext{
1 UNESCO, Convención sobre la protección del patrimonio Mundial, Cultural y Natural; el Comité del Patrimonio Mundial ha inscrito: Paisaje Agavero y Antiguas Instalaciones Industriales de Tequila en la Lista del Patrimonio Mundial. La Inscripción en esta lista confirma el valor excepcional y universal de un sitio cultural o natural que debe ser protegido para el beneficio de la humanidad. 16 de Julio de 2006. Texto expresado en la declaratoria.

Citación: ORENDAIN, T. E. y MICHEL, J. G. Paisaje biocultural y territorio, reflexiones sobre la gestión del patrimonio del "paisaje agavero y antiguas instalaciones del tequila". En: Libro de proceedings, CTV 2018. XII Congreso Internacional Ciudad y Territorio Virtual. "Ciudades y Territorios Inteligentes". UNCuyo, Mendoza, 5-7 septiembre 2018. Barcelona: CPSV, 2018, p. 63-76. 
Comité del Patrimonio Mundial como un ejemplo excepcional de la categoría patrimonial de Paisaje Biocultural Productivo, es decir, el paisaje natural transformado por el hombre durante cuatro centurias.

\section{El polígono de protección}

Éste está integrado por:

- Zona Núcleo 1; tiene una superficie territorial de 34,654.67 ha. Que es donde predomina el cultivo de agave azul, corresponde a la ladera de volcán de Tequila, parte del valle y la barranca del Rio Grande de Santiago localizado en los municipios del, Arenal, Amatitán y Tequila integrando los centros de población, además de parte del territorio de Magdalena sin incluir su asentamiento principal.

- Zona Núcleo 2; tiene una superficie territorial de 360.18 ha. corresponde a la delimitación del sitio arqueológico de Guachimontones, en el municipio de Teuchitlán.

- Área de Amortiguamiento con una superficie de 51,261.33 ha. donde predominando el Valle y la Barranca del Rio Grande de Santiago, en los municipios de Tala, Ahualulco de Mercado, San Juanito de Escobedo y Zapopan todos del Estado de Jalisco México.

\section{A doce años de la Declaratoria}

Paisaje Agavero y las Antiguas Instalaciones Industriales de Tequila, constituyó un momento apropiado para realizar una serie de reflexiones sobre el manejo que han realizado las instituciones en este Paisaje Biocultural que servirá para refrendar retos y compromisos acordes con los postulados de la UNESCO para una gestión eficaz.

Ello ha supuesto realizar un ejercicio institucional de evaluación que conlleva autocrítica, y una actitud proclive para reconocer debilidades, condición necesaria para superarlas. México, y Jalisco en particular, asumieron el reto de dar sentido patrimonial a un paisaje excepcional, irrepetible en otras partes del mundo. Tal desafío implica rediseñar los instrumentos de planificación urbana y territorial en términos innovadores que generen el equilibrio adecuado para la población que habita esta región de Jalisco, México.

Bajo tal consigna, y luego de casi dos años de trabajar en el diagnóstico y actualización del Plan de Manejo, de este Paisaje Biocultural Vivo, se presenta algunas reflexiones en la que se refuerzan acciones que se derivan del entendimiento del agave como símbolo querefrenda una arraigada tradición agraria, y que en conjunción con las instalaciones industriales articulan un territorio de gran personalidad geográfica y de oportunidades.

El carácter del paisaje se percibe a primera vista mediante el mosaico de parcelas pobladas de agave azul. Una línea de actuación, quizá la principal, consiste en trabajar de manera constante para que dicho legado mantenga firme su carácter, sea un digno exponente de la riqueza cultural del mundo, y contribuya a detonar procesos de desarrollo social y económico que se consoliden a través ofrecer oportunidad de permanecía y oportunidades dignas para la población que habita este territorio y la que emigra en busca de mejores oportunidades convirtiéndose en un territorio de oportunidades y de equilibrio con el medio ambiente.

Citación: ORENDAIN, T. E. y MICHEL, J. G. Paisaje biocultural y territorio, reflexiones sobre la gestión del patrimonio del "paisaje agavero y antiguas instalaciones del tequila". En: Libro de proceedings, CTV 2018. XII Congreso Internacional Ciudad y Territorio Virtual. "Ciudades y Territorios Inteligentes". UNCuyo, Mendoza, 5-7 septiembre 2018. Barcelona: CPSV, 2018, p. 63-76. 
Se plantea la conveniencia de intervenir dos ámbitos. El de la escala particular, es decir a nivel de parcela, donde prevalece en primera instancia la dimensión económica motivada por llevar el cultivo hasta su fase de industrialización, lo cual involucra la generación de empleo agrario y la salvaguarda de añejas tradiciones campesinas identificadas como patrimonio inmaterial.

Simultáneamente se requiere abordar la escala general, entendida como una política públicoprivada comandada por el Estado, y orientada, entre otros objetivos, a que el universo agrario inserto en el polígono protegido (zona núcleo 1), mantenga el agave como cultivo principal. Tal objetivo, además de relacionarse con el interés económico, se articula con la dimensión sociocultural dados los múltiples significados históricos y patrimoniales del territorio.

Esto porque de manera recurrente se presentan situaciones de crisis en el ámbito agrario: retracción de cultivos y abandono de parcelas, lo que se desprende del principio de incertidumbre derivado del ciclo de larga duración (siete años), para la maduración del agave y por tanto, resulta complicado alinear la oferta de agave listo para la cosecha con la demanda por parte de la industria. En los episodios críticos también han contribuido problemas de plagas que provocan fracaso económico para los agaveros agricultores. De acuerdo con esto, la búsqueda de un modelo sostenible denota una dimensión ambiental.

Por ello resulta fundamental asumir que estamos ante un paisaje biocultural y productivo en el que la búsqueda de equilibrio es permanente, el Paisaje de agave azul es un paisaje vivo y su comportamiento varía de acuerdo con reglas de la economía a través de la oferta y la demanda principalmente de la bebida llamada Tequila, mismas que pueden ser controladas con la aplicación de agricultura por contrato entre otras medidas. La estrategia para el Paisaje Agavero es ensamblar en forma equilibrada objetivos económicos, ambientales y culturales de cara a conservar in situ y a largo plazo la vigencia de un sistema productivo complejo que incluye el cultivo de agave y la fabricación del tequila.

Todo lo anterior requiere transitar de un enfoque monumentalista a un tratamiento paisajístico en constante cambio. Gestionar un paisaje biocultural productivo demanda habilidades distintas a las que exigen bienes patrimoniales más estáticos en donde la presencia del artesano en nuestro tiempo adquiere un rol importante y de constante cambio, y también se hace necesaria una cultura de cooperación interinstitucional y acuerdos con los agentes privados mayores beneficiarios del proceso de comercialización y con muy poco arraigo con el bien cultural.

A su vez, el tratamiento paisajístico demanda la identificación de un repertorio de tipologías territorializadas de paisaje, las cuales responden a una clasificación diversa y que a efectos planificadores se conciban de manera jerarquizada. Indudablemente el paisaje conformado por los campos de agave y las instalaciones industriales tequileras encabezan la lista de paisajes y también las prioridades en materia de conservación.

Entender el atributo de paisaje cultural productivo implica reconocer su dinamismo y por ello no debe esperarse una cobertura total de superficie de agave azul, e incluso aceptar que esa condición no es deseable ya que los monocultivos son sensibles a desarrollar plagas y enfermedades. La mixtura con cultivos tradicionalescomo el maíz, frijol, cacahuate y hasta con frutales principalmente en la ladera del volcán tequila y en la barranca del rio Grande Santiago, no debería verse como un aspecto negativo.

Tampoco es acertado esperar que el territorio agavero observe una frontera agrícola estática. El criterio sería que domine el agave bajo umbrales flexibles, el contorno de la superficie agave

Citación: ORENDAIN, T. E. y MICHEL, J. G. Paisaje biocultural y territorio, reflexiones sobre la gestión del patrimonio del "paisaje agavero y antiguas instalaciones del tequila". En: Libro de proceedings, CTV 2018. XII Congreso Internacional Ciudad y Territorio Virtual. "Ciudades y Territorios Inteligentes". UNCuyo, Mendoza, 5-7 septiembre 2018. Barcelona: CPSV, 2018, p. 63-76. 
azul será siempre elástico. La prueba de eficiencia será la observancia de una calidad del paisaje agavero como paisaje Biocultural productivo, rentable en términos económicos, y libre de agentes patógenos. Si se consigue tal condición, unida al atributo de dominancia, se garantizará una imagen paisajística acorde con la filosofía conservacionista y se asegurará su papel como territorio atractivo para el goce estético y recreativo de ciudadanos locales y turistas.

La preservación del Paisaje Agavero demanda esfuerzos por sincronizar el sistema agavetequila y una labor permanente de monitoreo en la que se diagnostiquen episodios de estabilidad, crecimiento o regresión y se tomen las medidas que se consideren adecuadas para asegurar la imagen paisajística esperable en un sitio catalogado como Patrimonio de la Humanidad.

Desde una perspectiva integral las tipologías de paisaje pueden tener su símil como unidades de paisaje organizadas geográficamente a partir de las tres grandes morfoestructuras 0 dominios territoriales: los Valles agaveros coincidentes principalmente con la Zona Núcleo 1, y las otras dos que forman parte del dominio de la Zona de Amortiguamiento: el Complejo Volcánico de Tequila y una parte del Cañón del Río Grande Santiago.

Además, se añade la Zona Núcleo 2 donde prevalece el patrimonio arqueológico representado por Los Guachimontones.

Los contenidos patrimoniales en cada una de esas tres grandes unidades, además de revelar una gran riqueza y diversidad, tendrán la función de diseñar el sistema de jerarquías o prioridades dentro del Plan de Manejo. Como función añadida, permitirán comunicar el sistema de competencias por parte de las diferentes instancias de la administración pública en sus tres niveles de gobierno.

La amplitud del perímetro declarado Patrimonio Cultural de la Humanidad y la diversidad de sus contenidos demandan un modelo de gestión que no puede desarrollarse bajo una lógica de fragmentación político-administrativa que favorece la dispersión de acciones o los bloqueos. Esta condición impide además la visión de largo plazo, por lo que se torna prioritario atender ese tema.

Existe un Organismo legislativo fundado en 2008 que es; La Comisión para la Conservación, Protección, Revalorización, Rehabilitación y Difusión del Paisaje Agavero y Antiguas Instalaciones de Tequila Jalisco, es coordinada por la Secretaría de Cultura, misma que de acuerdo al artículo Tercero, fracción II del Acuerdo de creación de la Comisión, actúa como Secretario Ejecutivo.

Dado que la gestión del perímetro declarado debe estar sometida a un ejercicio de evaluación permanente, en el que se expliciten y sean verificables indicadores que revelen si las condiciones del sitio evolucionan positivamente, habrá que decir que la curva de aprendizaje obtenida a lo largo de ya más de doce años, corrobora que la gestión es débil dado que es más legislativa que operativa, cuando los esfuerzos han sido esporádicos y $\sin$ recursos establecidos.

Resulta necesario que la Comisión atienda los procesos de gestión en el día a día, con el objeto de lograr las metas establecidas en el Plan de Manejo.

Citación: ORENDAIN, T. E. y MICHEL, J. G. Paisaje biocultural y territorio, reflexiones sobre la gestión del patrimonio del "paisaje agavero y antiguas instalaciones del tequila". En: Libro de proceedings, CTV 2018. XII Congreso Internacional Ciudad y Territorio Virtual. "Ciudades y Territorios Inteligentes". UNCuyo, Mendoza, 5-7 septiembre 2018. Barcelona: CPSV, 2018, p. 63-76. 


\section{4. objetivo principal del plan de manejo del sitio}

La imagen objetivo será diferencial: la Zona del Volcán Tequila (entre las cotas 1500 y los 2900 metros sobre el nivel del mar), sujeta a un tratamiento como política de protección como paisaje natural, en particular el bosque templado; mientras que en los valles Agaveros y en la zona cañera del otro lado del volcán tequila en el municipio de Tehuchitlán donde se ubican los Guachimontones (entre 1100 y 1500 msnm), es decir en las zonas núcleo 1 y 02, prevalece su estructuración como paisaje cultural antropizado; y el Cañón del Río Grande Santiago (entre 600 y $1100 \mathrm{msnm}$ ), donde se produce una mixtura como paisaje cultural y natural, este último constituido por un bosque tropical caducifolio sujeto en un tramo a una política de conservación.

Este planteamiento territorial se articula para su gestión y manejo en seis ejes sectoriales, correspondientes a los patrimonios, cada uno con paisajes particulares, que integran el Sitio y que conviene proteger diferencialmente:

a. Patrimonio Natural. Se refiere a los componentes geomorfológicos, de cobertura vegetal, fauna y recursos acuíferos, de los cuales resultan paisajes naturales.

b. Patrimonio Rural. Formado principalmente por el paisaje agavero, esto es por un paisaje cultural productivo (PCP); terracerías histórica, infraestructura territorial, líneas ferroviarias y bordos.

c. Patrimonio Arqueológico. Compuesto por zonas, elementos y vestigios de la ocupación prehispánica del territorio.

d. Patrimonio Inmaterial. Integrada por costumbres, tradiciones, gastronomía, identidades regionales, fiestas y leyendas. Se considera también el reconocimiento de la existencia de una dinámica, con un sentido de uso y apropiación del espacio, a través del cual se configura y robustece el tejido social local de los asentamientos humanos más representativos de la región agavera como son Arenal, Amatitán, Tequila y Magdalena en la zona núcleo 1, además de la zona de amortiguamiento del otro lado del volcán Tequila donde se ubican los municipios de Tala, Tehuchitlán, Ahualulco del Mercado y San Juanito de Escobedo.

e. Patrimonio Urbano. Se refiere a la morfología, la traza y el paisaje urbano, como rasgos de singularidad de las diferentes poblaciones.

f. Patrimonio Arquitectónico. Está compuesto por las antiguas instalaciones productoras del tequila, tales como tabernas, fábricas y haciendas; así como otras tipologías de inmuebles de valor histórico y artístico.

La política cultural del Paisaje Agavero girará en torno a la conservación del equilibrio entre el medio natural y el medio sociocultural; de los valores asociados con la arqueología y la arquitectura; de la visibilizarían y puesta en valor de los saberes locales y técnicas de cultivo del agave, además de las expresiones culturales que se dan entorno a una cultura de incursión donde la planta del agave eta más presente que nunca como un valor agregado; con la intención de fortalecer procesos de desarrollo, en congruencia con las recomendaciones de la UNESCO.

Citación: ORENDAIN, T. E. y MICHEL, J. G. Paisaje biocultural y territorio, reflexiones sobre la gestión del patrimonio del "paisaje agavero y antiguas instalaciones del tequila". En: Libro de proceedings, CTV 2018. XII Congreso Internacional Ciudad y Territorio Virtual. "Ciudades y Territorios Inteligentes". UNCuyo, Mendoza, 5-7 septiembre 2018. Barcelona: CPSV, 2018, p. 63-76. 


\section{Fundamentos generales de apoyo para la protección y salvaguarda del patrimonio}

- Conservar y proteger las características del medio físico natural en las inmediaciones del volcán, los valles y la barranca dentro de la Declaratoria.

- Conservar, fomentar y poner en valor el patrimonio rural con una visión sostenible para fortalecer la identidad desde lo local.

- Proteger y conservar el patrimonio arqueológico localizado en la zona declarada.

- Recuperar y proteger el patrimonio inmaterial en la zona del Paisaje Agavero, principalmente la que tienda a recuperar la memoria de trasmisión del conocimiento y su evolución de cara a la innovación y adecuación a los nuevos tiempos.

- Proteger, recuperar y conservar la autenticidad de los centros de población para mantener la imagen urbana tradicional sin menos cabo de la transformación contemporánea como garante del proceso de evolución.

- Proteger, recuperar y restaurar la arquitectura típica y los monumentos, dentro y fuera de los centros de población.

\section{Estrategias para consolidar el Paisaje Biocultural Vivo}

\subsection{Patrimonio rural}

- Documentar las prácticas culturales y agrícolas de las localidades rurales que en su dinámica cotidiana contribuyen en la configuración, apropiación y consolidación del paisaje agavero como un paisaje biocultural productivo.

- Alinear las políticas públicas para fomentar apoyos especiales para el polígono de protección para la recuperación de la agricultura, para la siembra tradicional del Agave Azul y/o tecnificada, con base en los usos y costumbres regionales en la zona núcleo 1 determinado por la UNESCO.

- Promover el aprovechamiento sostenible con base en el sistema de siembras complementarias (Maíz, Calabaza, Frijol y Árboles frutales), con el uso de fertilizantes y control de plagas orgánicos. Así como el cultivo alternado y el descanso de tierras.

- Promover rutas creativas; vías verdes, con un enfoque de agroturismo cultural o de turismo consciente de las características rurales propias de la zona deAgave Azul.

- Fomentar los estudios científicos sobre el patrimonio de Agave Azul de lacomarca.

- Promover usos alternativos de la planta de agave, así como de los derivadosdel proceso de destilación, por ejemplo, para la elaboración de artesanías, manualidades, abonos.

Al cambio de centuria el paisaje de Agave Azul continúa llamando la atención de nacionales y extranjeros. Esta situación es testimoniada por el viajero inglés T. Phillip Ferry, quien en 1909

Citación: ORENDAIN, T. E. y MICHEL, J. G. Paisaje biocultural y territorio, reflexiones sobre la gestión del patrimonio del "paisaje agavero y antiguas instalaciones del tequila". En: Libro de proceedings, CTV 2018. XII Congreso Internacional Ciudad y Territorio Virtual. "Ciudades y Territorios Inteligentes". UNCuyo, Mendoza, 5-7 septiembre 2018. Barcelona: CPSV, 2018, p. 63-76. 
escribe: "Esta planta es un poco más pequeña que la productora del pulque, con hojas angostas y un color azulado. Esta crece mejor sobre la pendiente de una montaña llamada El Cerro de Tequila, en el distrito del mismo nombre."

Además, contrario a la idea de que el maguey sólo era plantado y cosechado, una lectura acuciosa de la información histórica, además de los estudios realizados en las últimas dos décadas del siglo $\mathrm{XX}$, refieren cómo desde siempre la planta ha sido cultivada con una variedad de técnicas e instrumentos, poniendo en práctica saberes tradicionales a la par de adaptaciones e innovaciones derivadas de investigaciones tendientes a mejorar rendimientos, disminuir costes y facilitar las condiciones en las cuales se realiza el trabajo.

\subsection{Patrimonio inmaterial}

- Fomentar los estudios culturales para la salvaguardia sobre el patrimonio inmaterial de la zona de protección del paisaje agavero.

- Documentar las prácticas colectivas relacionadas con el agave, su cultivo y aprovechamiento, por ejemplo, en la gastronomía, en los actos festivos, religiosos o rituales para garantizar la recuperación de la identidad desde lo local.

- Documentar el conocimiento ancestral sobre los distintos sistemas de siembra, cultivo y cosecha del agave.

- Promover los valores, las prácticas y las tradiciones culturales, además del fomento del conocimiento desarrollado en torno al cultivo de agave y sus derivados.

José María Najar, escribe en su Nueva Geografía del Estado de Jalisco sobre la expansión de los cultivos sobre el territorio: ...sobre todo en el último cantón (forma de delimitación impuesta por los españoles en la nueva España), en que se ven inmensas mezcaleras de vino cuyos límites no alcanzan la vista y en este cantón el giro principal es la elaboración de alcohol de mezcal, conocido generalmente con el nombre de vino tequila. Con igual nombre se conoce el que se elabora en el de Sayula y Ameca, aunque en los tres cantones, toman, además, el nombre de la hacienda o fabrica que lo produce.

Es importante tener presente que un paisaje productivo continuamente está siendo intervenido por el humano es por eso que se usa ahora el termino Biocultural, porque se está rehaciendo contantemente y manteniendo por lo mismo se justifica el que se hable de un paisaje vivo. En ese sentido, para llegar al momento de la jima (el corte), primero hay que pasar por la plantación de los hijuelos seleccionados en terrenos que previamente fueron preparados para ello.

El conocimiento de la estructura y composición del suelo es uno de los adelantos científicos de años anteriores a la Declaratoria del Paisaje Agavero. Ello ha permitido mejorar las características de las tierras al momento de iniciar una plantación, sea a través de la neutralización del PH así como del enriquecimiento con abonos.

Después de ello vendrán varios años de mantenimiento de los agaves, (siete años para que madure), mediante escardas, aplicación de fertilizantes para cierto tipo de plantaciones, deshierbes, control de plagas, desahíje y nivelación de terrenos en caso de erosión o deslaves.

Citación: ORENDAIN, T. E. y MICHEL, J. G. Paisaje biocultural y territorio, reflexiones sobre la gestión del patrimonio del "paisaje agavero y antiguas instalaciones del tequila". En: Libro de proceedings, CTV 2018. XII Congreso Internacional Ciudad y Territorio Virtual. "Ciudades y Territorios Inteligentes". UNCuyo, Mendoza, 5-7 septiembre 2018. Barcelona: CPSV, 2018, p. 63-76. 
Otras actividades del campo fincadas en los usos tradicionales son la limpieza cíclica de la labor que de acuerdo con la zona recibe el nombre de parcela, solar o mezcalillera; el "barbeo" o despunte de las pencas para acelerar la producción de azúcares en la planta madura, la detección de los ejemplares "anovillados" que anuncian la aparición del "quiote" o floración en las plantas maduras. La última actividad que corona ese esfuerzo de años es la jima o corte de las pencas del agave para dejar al descubierto la piña del agave que es el fruto final.

Lo mismo debe mencionarse con respecto a los métodos de selección, plantación, cultivo y cosecha del agave, los cuales se han ido mejorando constantemente, creándose una cultura rural que aún es posible identificar por sus múltiples expresiones y prácticas culturales en toda la región.

Otra de las expresiones del mestizaje se encuentra en el proceso de fermentación y destilación. El primero ya practicado por los indígenas, el segundo posible gracias a la introducción del alambique de origen árabe en el siglo XVI. Más tarde, con el desarrollo de la minería la comarca tendría un desarrollo importante, que se reflejaría en la asociación entre la bebida y la localidad productora.

Por ello puede sostenerse que el diseño y configuración actual de las plantaciones y destilerías comprendidas dentro del Paisaje Agavero es resultado de un largo proceso de decantación de experiencias, surgidas del ensayo y el error en las prácticas de cultivo, y de procesos de manufactura de una gran diversidad de productos todo este proceso tradicional que se ha trasmitido de generación en generación a lo largo de cuatro siglos por los habitantes de la región lo que lo convierte en un legado vivo de valor universal excepcional irrepetible en el mundo.

\subsection{Patrimonio urbano}

- Mantener, conservar y adaptar la imagen urbana a la tipología tradicional de los municipios de la zona núcleo 1, como parte del patrimonio integrado a través de los instrumentos de planeación urbano y territorial vigentes.

- Fomentar los estudios relacionados con el valor de los centros históricos y polígonos de protección en términos urbanísticos de conservación, preservación y de intervención para la adaptación a los nuevos retos que demandan esta sociedad en constante cambio.

\subsection{Patrimonio arquitectónico}

- Conservar los monumentos arquitectónicos importantes de los centros urbanos comprendidos en el polígono de protección del Paisaje Agavero.

- Identificar y restaurar los monumentos arquitectónicos de las zonas rurales del Paisaje Agavero.

- Consolidar la arquitectura patrimonial adquirida por las entidades Gubernamentales y de Particulares al servicio de la comunidad para el desarrollo de la cultura, atendiendo a sus valores arquitectónicos y usos sociales.

- Promover los estudios relativos al valor del patrimonio arquitectónico del Paisaje Agavero.

Citación: ORENDAIN, T. E. y MICHEL, J. G. Paisaje biocultural y territorio, reflexiones sobre la gestión del patrimonio del "paisaje agavero y antiguas instalaciones del tequila". En: Libro de proceedings, CTV 2018. XII Congreso Internacional Ciudad y Territorio Virtual. "Ciudades y Territorios Inteligentes". UNCuyo, Mendoza, 5-7 septiembre 2018. Barcelona: CPSV, 2018, p. 63-76. 
- Promover el turismo cultural en los sitios de monumentos arquitectónicos públicos y privados atendiendo a la parte social, dentro del Paisaje Agavero.

\section{Ejemplos de la evolución en el tiempo del Patrimonio Inmaterial en los Centros de Población del Paisaje Agavero}

\subsection{Manifestaciones Religiosas en Arenal}

Las festividades patronales se celebraban en la capilla de La Parreña, el 2 de febrero, día de La Candelaria. Originalmente era la fiesta tradicional, a la advocación de la Virgen del Rosario, y se celebraba del 25 de enero al 2 de febrero.

El nombre original de la parroquia es Virgen del Rosario Patrona de Arenal, pero recientemente se cambió a Nuestra Señora del Rosario de Talpa de Arenal. Es decir, en el pueblo se tiene dos imágenes de vírgenes con nombres semejantes. Por ello se celebra a la Virgen de Talpa, cuya fiesta en realidad está fechada el 7 de octubre. Esta última se caracteriza por la peregrinación donde los hombres cargan cañas de maíz de la cosecha reciente, sin embargo, como en ocasiones para esa primera semana de octubre todavía no hay cosecha, se cambió para enero.

El punto de reunión suele ser la Antigua Hacienda de La Calavera, hoy Museo Interpretativo del paisaje agavero. Las calles por donde va a pasar la Virgen se adornan con papel o plástico. Mientras las personas llevan las cañas con las mazorcas, y las mujeres flores de Santa María, quienes peregrinan en tractores los cargan de pencas de agave (desde 2008), con la finalidad de agradecer la cosecha del año. El momento culminante de la celebración es la misa. Para solventar los gastos de la fiesta se realiza una colecta en todo el municipio, la cual es organizada por el comisariado ejidal.

\subsection{Leyenda de Tequila de Mayahuel}

Entre las leyendas que cuentan algunos lugares y acontecimientos de Tequila, se dice que Huaxicar protegía a una mujer que con mezcal curaba enfermedades, por lo que se considera que ella fue quien descubrió la bebida de mezcal conocida como tequila. Esta mujer de quien no se conoce su nombre la llamaron Mayahuel.

\subsection{Leyenda de Amatitán}

Una de las más conocidas leyendas es la de unos indígenas nahuatlatas, que fueron de los primeros pobladores del lugar, vieron caer del cielo un rayo sobre una planta de agave y cuando se acercaron a él se dieron cuenta de que le escurría miel que los satisfizo; a raíz de este suceso los indígenas comenzaron a cocer los agaves bajo tierra que luego de macerarlos y posteriormente fermentarlos, se obtenía el preciado licor.

Un asunto importante a tener en cuenta es que el patrimonio inmaterial al estar vinculado con sus portadores, es un elemento vivo y vivificante, en constante transformación. De esta forma también cabe decir que experimenta cambios, y si bien en algunos momentos está fuertemente vinculado con el pasado, en otros casos se trata de nuevas formas de relacionarse con el entorno, pero también de estrategias de adaptación a los nuevos contextos.

Por ejemplo, en Tequila y Amatitán al menos desde la época colonial, el bagazo caliente

Citación: ORENDAIN, T. E. y MICHEL, J. G. Paisaje biocultural y territorio, reflexiones sobre la gestión del patrimonio del "paisaje agavero y antiguas instalaciones del tequila". En: Libro de proceedings, CTV 2018. XII Congreso Internacional Ciudad y Territorio Virtual. "Ciudades y Territorios Inteligentes". UNCuyo, Mendoza, 5-7 septiembre 2018. Barcelona: CPSV, 2018, p. 63-76. 
desechado por las tabernas y antiguas instalaciones era aprovechado por ganado mayor y rumiantes quienes consumían algunas porciones debido a la cantidad de azúcares que todavía contenían esos restos. Es fácil imaginar que se trataba de una época en la cual las instalaciones fabriles no eran construcciones cerradas, preocupadas por la inocuidad, y donde por ende podían encontrarse corrales y hasta pequeñas granjas ligadas a las tabernas o por donde podían cruzar animales atraídos por el aroma del agave cocido y por el recién destilado.

También durante mucho tiempo la fibra del bagazo era aprovechada por los ladrilleros para la elaboración de tabiques con una consistencia particular, precisamente por la argamasa que se lograba al mezclar diferentes tipos de tierra con el bagazo todavía húmedo.

En la actualidad, en relación directa con la bonanza que experimenta la industria tequilera y la atracción de turistas que va en aumento para la comarca distinguida por la UNESCO como Paisaje Biocultural Patrimonio de la Humanidad, han emergido otro tipo de formas de aprovechamiento de esos residuos, los cuales de alguna manera se atan al patrimonio inmaterial heredado por generaciones, puesto que hay cierta recuperación de tecnologías de antaño, pero no dejan de tener su lado novedoso sobre todo por las potencialidades que otro tipo de herramientas, procesamientos y dinámicas de la vida moderna permiten.

Así, encontramos que en la actualidad ese mismo bagazo desechado por la industria tequilera es reutilizado para la elaboración de papel de agave, de diferentes calidades, es el caso por ejemplo de una pequeña empresa familiar, ubicada en el patio trasero que antaño fuera una huerta y corral de una casona de Tequila. Ahí una pareja e hijos más algunos empleados temporales combinan técnicas antiguas de elaboración de papel, con tecnologías y materiales recientes. El resultado es un producto ecológico bien valorado por los conocedores de este nicho de mercado.

A su vez en el proceso de elaboración del papel éste desecha una "sustancia" que al secar tiene una fisonomía plástica, con la que se elaboran utensilios de cocina; del bagazo de agave se produce también hilo, en diferentes gruesos para ser tejido a gancho, o hilado; de estos textiles existen ejemplos en Oaxaca, los conocidos telares de cintura para la elaboración de rebozos, o la "pita" traída a Jalisco, la cual en Ocotlán trabajan los talabarteros sobre el cuero, que se realiza al bordar con hilo fino, cardado de las pencas de otro tipo de agave, para la elaboración de variadas artesanías. Éstas combinan diseños como el bordado, calado, cincelado, pirograbado; en productos como cintos, sombreros, monturas, huaraches, bolsos y joyería con gran creatividad; así como en la indumentaria del traje de charro y otros elementos usados en la charrería.

Es decir, la bonanza tequilera y el reconocimiento del Paisaje Agavero ha creado condiciones para la aparición de diferentes alternativas artísticas y artesanales regionales, las cuales a su vez han detonado, a través de su articulación, el desarrollo de otras industrias y proyectos empresariales, como el caso del bagazo- papel-utensilios de plástico, convirtiendo residuos en subproductos, pero además enlazando emprendedores en diferentes partes del país.

El municipio de Amatitán destaca por la destreza de las manos de mujeres que creativamente deshilan servilletas que adornan los muebles de las casas. Las principales materias primas para las artesanías locales son el cuero y los cuernos de bovinos, no obstante, a pesar de su reconocimiento estatal, la escasa difusión es un problema para la ampliación de este sector.

Citación: ORENDAIN, T. E. y MICHEL, J. G. Paisaje biocultural y territorio, reflexiones sobre la gestión del patrimonio del "paisaje agavero y antiguas instalaciones del tequila". En: Libro de proceedings, CTV 2018. XII Congreso Internacional Ciudad y Territorio Virtual. "Ciudades y Territorios Inteligentes". UNCuyo, Mendoza, 5-7 septiembre 2018. Barcelona: CPSV, 2018, p. 63-76. 


\section{Conclusiones}

Producto del diagnóstico, para preservar el patrimonio inmaterial y propiciar oportunidades que permitan la permanencia de la población en su lugar de origen:

El carácter casero es una alusión al espacio en el cual se materializan muchas de las ideas surgidas a partir de la necesidad, y que luego serán validadas en campo tanto en su aplicación como por el resto de compañeros de trabajo. En ese sentido, la experiencia nutre los conocimientos previos y las respuestas ante circunstancias semejantes volviendo la actividad tradicional. También definirla como "casera" es para hacer una referencia a que esas creaciones son propiedad de los sujetos.

Por último, la analogía con las artesanías es triple. Primero por tratarse de piezas únicas, concreción material de una necesidad satisfecha de esa manera particular; segundo porque durante todo el proceso participa el mismo trabajador que usará el instrumento en sus labores agrícolas, y tercero porque representa la corta distancia existente entre el trabajador, la planta y el entorno en donde crece. Una herramienta elaborada en China, comprada en una tienda especializada, homogeneiza el desempeño de los trabajadores, y amplía la distancia entre los saberes locales y el espacio donde se territorializan, lo cual no sucede cuando se ponen en acción conocimientos y técnicos tradicionales, las cuales han mostrado su efectividad.

Lo anterior es un ejemplo de la importancia de revalorizar el trabajo artesanal que se ha realizado históricamente para dar vida al paisaje cultural productivo, sustento del Paisaje Agavero patrimonio de la humanidad. Desde la reproducción de los mejores ejemplares, la preparación del terreno, las labores culturales o cultivos realizados, hasta el final del mantenimiento cuando llega la jima, hay un valioso conjunto de experiencias, saberes locales, maneras de hacer que son medulares al crear los paisajes regionales, y por ello es importante documentar constantemente lo que sucede en los campos Agaveros con los portadores de ese patrimonio. La consigna es que mejores condiciones de salud tengan los trabajadores, más se pongan en práctica los saberes artesanales y tradicionales, mejores serán las condiciones en las que se encuentren las plantaciones agaveras.

Poner énfasis en la importancia de las prácticas culturales profundas que han dado vida al paisaje agavero, puede ser un factor para atender algunos de los problemas regionales derivados de la tercerización que se experimenta y del abandono de las actividades primarias por diferentes cuestiones.

Otros asuntos que deben tenerse en cuenta, y que se relacionan directamente con los datos expuestos en el apartado anterior tienen que ver con el desempleo, el analfabetismo, la migración por falta de oportunidades de crecimiento personal y laboral en la región. Uno más tiene que ver con la promoción de actividades que fomenten la convivencia social y una identidad regional.

Ahora, en el caso particular del municipio de El Arenal, la declaratoria del Paisaje Agavero parece tener poco impacto en términos culturales y sociales, sin embargo, es un municipio con una de las mayores transformaciones sociales en las últimas décadas, a causa de varios fenómenos socioeconómicos. Uno de ellos es la migración, de la cual ya se presentaron algunas cifras.

Citación: ORENDAIN, T. E. y MICHEL, J. G. Paisaje biocultural y territorio, reflexiones sobre la gestión del patrimonio del "paisaje agavero y antiguas instalaciones del tequila". En: Libro de proceedings, CTV 2018. XII Congreso Internacional Ciudad y Territorio Virtual. "Ciudades y Territorios Inteligentes". UNCuyo, Mendoza, 5-7 septiembre 2018. Barcelona: CPSV, 2018, p. 63-76. 
Una parte de los arenalenses viven de las remesas que sus paisanos y familiares envían, y con quienes mantienen una fuerte relación económica. Muchos de estos migrantes radican en California.

Los impactos culturales de esta migración, que ha llevado a muchos integrantes de las familias a vivir a Estados Unidos, no han sido aún evaluados. Sin embargo, los pobladores los reconocen $y$, al realizar una visita por algunas casas del centro poblado, pueden verse influencias en la vida diaria: adecuaciones en viviendas que van desde la transformación de los espacios cotidianos y los gustos arquitectónicos hasta el uso de electrodomésticos que no son comunes en los demás hogares mexicanos. Por supuesto que esto no es algo completamente asimilado ni extendido a toda la población, pero sí señala una influencia cultural fuerte. Para contar con detalles puntuales, habría que evaluar las transformaciones desde otras perspectivas para poder tener una idea clara de sus resultados sociales y culturales.

Todo ello da cuenta de la importancia de que desde la Comisión del Paisaje Agavero se suma la tarea de identificar, rescatar, poner en valor y difundir el valor del patrimonio cultural del Sitio, y con su capacidad de convocatoria, de involucrar a los diferentes actores de la región.

Citación: ORENDAIN, T. E. y MICHEL, J. G. Paisaje biocultural y territorio, reflexiones sobre la gestión del patrimonio del "paisaje agavero y antiguas instalaciones del tequila". En: Libro de proceedings, CTV 2018. XII Congreso Internacional Ciudad y Territorio Virtual. "Ciudades y Territorios Inteligentes". UNCuyo, Mendoza, 5-7 septiembre 2018. Barcelona: CPSV, 2018, p. 63-76. 